COMMUNICATIONS IN

ANALYSIS AND GEOMETRY

Volume 12, Number 5, 1039-1053, 2004

\title{
Global and Local Volume Bounds and the Shortest Geodesic Loops
}

\author{
StÉPHANe SABOURAu
}

\section{Introduction.}

The relations between the volume of complete Riemannian manifolds and the length of their shortest nontrivial geodesic loops under no curvature assumption are studied in this paper. We present and compare two lower bounds on the global volume, one of which admits a local version.

Previous curvature-free estimates have been first obtained with the injectivity radius. Namely, M. Berger proved in [3] the isoembolic theorem

$$
\operatorname{Vol}(M) \geq C_{n} \operatorname{inj}(M)^{n}
$$

for all complete Riemannian manifolds with a sharp positive constant $C_{n}$.

A local version was then established by C. Croke in [4]

$$
\operatorname{Vol} B\left(x_{0}, R\right) \geq C_{n} R^{n} \text { for all } R \leq \frac{1}{2} \operatorname{inj}(M)
$$

for all complete Riemannian manifolds but with a nonsharp constant.

Replacing the notion of injectivity radius with one of local geometric contractibility, M. Gromov extended M. Berger's global volume estimate in [7]. In the same way, an extension of C. Croke's local version was then established by R. Greene and P. Petersen in [6].

Other results have been obtained by M. Gromov and C. Croke, who compared the volume with the length of the shortest nontrivial closed geodesic, noted $\operatorname{scg}(M)$. In [7], M. Gromov proved that every 1-essential closed Riemannian manifold $M$ satisfies

$$
\operatorname{Vol}(M) \geq C_{n} \operatorname{scg}(M)^{n}
$$

for some positive constant $C_{n}$ depending only on the dimension $n$ of $M$.

Recall that an $n$-dimensional manifold $M$ is by definition $k$-essential if there exists a continuous map $\psi: M \longrightarrow K$ into a $K(\pi, k)$ space such 
that $\psi_{*}[M] \neq 0$ where $[M] \in H_{n}(M)$ is the fundamental class of $M$. The homology coefficients are in $\mathbb{Z}$, if $M$ is orientable, and in $\mathbb{Z}_{2}$, otherwise.

For the two-dimensional sphere, the previous inequality still holds as it was proved by C. Croke in [5]. These two statements are the only results known in this direction and no counter-example exist so far.

Throughout this paper, we will consider geodesic loops rather than closed geodesics. By definition, a geodesic loop (based at a point $*$ ) is a geodesic arc with endpoints $*$. In particular, a closed geodesic is a geodesic loop whose tangent vectors at its endpoints agree. The length of the shortest nontrivial geodesic loop of a Riemannian manifold $M$ is denoted by $\operatorname{sgl}(M)$.

The first theorem we will prove provides lower bounds on the diameter, on the volume of the whole manifold and on the volume of sufficiently small balls.

Theorem A. Let $M$ be a complete Riemannian manifold. Then there exists positive constant $C_{n}, C_{n}^{\prime}$ and $C_{n}^{\prime \prime}$ depending only on the dimension $n$ of $M$ such that

$$
\begin{gathered}
\operatorname{Vol}(M) \geq C_{n} \operatorname{sgl}(M)^{n} \\
\operatorname{Diam}(M) \geq C_{n}^{\prime} \operatorname{sgl}(M) \\
\operatorname{VolB}\left(x_{0}, R\right) \geq C_{n}^{\prime \prime} R^{n}
\end{gathered}
$$

for every ball $B\left(x_{0}, R\right)$ of radius $R \leq \frac{1}{2} \operatorname{sgl}(M)$ in $M$.

Explicit formulas for $C_{n}, C_{n}^{\prime}$ and $C_{n}^{\prime \prime}$ can be calculated. Note that $\operatorname{scg}(M)$ may be greater than $\operatorname{sgl}(M)$ and yield no universal lower bound on the volume of "small" balls of $M$. A counter-example is given by any Riemannian manifold on which a long and thin spike is glued.

The following result, introducing the lengths of geodesic double loops, refines the global volume estimate for 2-essential manifolds but does not admit any local version.

Theorem B. Let $M$ be a 2-essential complete Riemannian manifold. Then there exists positive constants $c_{n}$ and $c_{n}^{\prime}$ depending only on the dimension $n$ of $M$ such that

$$
\begin{gathered}
\operatorname{Vol}(M) \geq c_{n} \ell_{0}(M)^{n} \\
\operatorname{Diam}(M) \geq c_{n}^{\prime} \ell_{0}(M)
\end{gathered}
$$

where $\ell_{0}(M)$ is the length of the shortest double loop $\Phi_{0}$ among the double loops formed of two nontrivial geodesic loops of the same length based at the same point. 
The two geodesic loops which compose $\Phi_{0}$ may agree. In this case, since $\Phi_{0}$ is minimal, this latter reduces to a closed geodesic run twice and we get lower bounds on the volume and the diameter in terms of $\operatorname{scg}(M)$. Unfortunately, even after slight perturbations of the metric, the double loop $\Phi_{0}$ might still be composed of two distinct geodesic loops. This will be discussed in more details at the end of this paper.

The example of a long and thin cone glued to a Riemannian manifold shows that $\ell_{0}(M)$ provides no universal lower bounds on the volume of "small" balls. This shows the difference in nature between the shortest geodesic loop and the double loop $\Phi_{0}$. Note that considering multi-loops formed of $k$ nontrivial geodesic loops of the same length based at the same point (with $k$ fixed) instead of double loops does not really change the problem since their shortest representants have comparable lengths.

In [1], M. Anderson proved a compactness theorem for metrics whose Ricci curvature, diameter, volume and length of the shortest geodesic loop are bounded. From Theorem A, it turns out that the required bound on the volume can be dropped. In [14], an upper bound similar to the second inequality of Theorem A has also been established by R. Rotman for simply connected Riemannian manifolds with nontrivial second homology group and with nonnegative curvature. These topological and geometrical assumptions on the manifold can actually also be dropped.

Similar curvature-free estimates were independently obtained in [12] by A. Nabutovsky and R. Rotman who found upper bounds on the minimal mass of nontrivial stationnary one-cycles (see also [11] for estimates depending on the curvature).

In the first section, we present a map extension criterion based on a simplex filling process and derive Theorem A using R. Greene and P. Petersen's theorem (see [6]). In the second section, we introduce a variational calculus on the double loop space and describe the structure of critical points. Then, we prove Theorem B using filling radius estimates developped by M. Gromov in $[7]$.

The author would like to thank I. Babenko and C. Croke for several helpful conversations and comments.

\section{Extension process and short geodesic loops.}

In this part, we construct a map extension process and prove Theorem A. 


\subsection{Preliminaries.}

The notion of retraction of loop spaces and the definition of ordered complex are presented in this section.

Let $(X, d)$ be a metric space, more simply noted $X$, and $*$ be a point in $X$. The loop space of $X$ based at $*$, noted $\Omega_{*} X$, is by definition the space of continuous loops $\gamma:[0,1] \longrightarrow X$ with endpoints $*$. It is endowed with the $C^{0}$-topology. Let $\kappa \geq 0$, we define $\Omega_{*}^{\kappa} X$ as the space of loops based at $*$ of length less than $\kappa$. The length of a loop $\gamma$ of a metric space is given by

$$
L(\gamma)=\sup \left\{\sum_{i=0}^{m-1} d\left(\gamma\left(s_{i}\right), \gamma\left(s_{i+1}\right)\right) \mid\left(s_{i}\right)_{0 \leq i \leq m} \text { subdivision of }[0,1]\right\}
$$

The Birkhoff curve-shortening process with fixed endpoints is defined on the loop space of Riemannian manifolds (cf. [10] for a precise definition). It gives rise to a continuous length non-increasing homotopy $\gamma_{t} \in \Omega_{*} M$ from a fixed loop $\gamma \in \Omega_{*} M$ to a geodesic loop based at $*$. Furthermore, geodesic loops are the only fixed points of this process. Therefore, if $\kappa<\operatorname{sgl}(M)$, there exists no nontrivial critical point for the length functional in $\Omega_{*}^{\kappa} X$. In this case, the Birkhoff process gives rise to a retraction from any loop space $\Omega_{*}^{\kappa} X$ into the singleton formed by the point curve $*$.

This property of retraction of loop spaces can also be set out for general metric spaces. Furthermore, it may be weakened, requiring that only finite dimensional subspaces of the loop space be retractible. In the rest of this part $\kappa_{0} \geq 0$ and $m \in \mathbb{N}$ are fixed and the metric spaces $X$ considered satisfy the following property :

For every point $* \in X$, every continuous map $f: P \longrightarrow \Omega_{*}^{\kappa_{0}} X$ defined on any simplicial complex of dimension $\leq m$, there exists a continuous map $F: P \times[0,1] \longrightarrow \Omega_{*}^{\kappa_{0}} X$ such that

- $\quad F$ agrees with $f$ on $P \times\{0\}$

- $F$ reduces to the constant loop $*$ on $P \times\{1\}$

- $L\left(\gamma_{p, t}\right) \leq L\left(\gamma_{p, s}\right)$ whenever $0 \leq s \leq t \leq 1$ where $\gamma_{p, t}=F(p, t)$.

An ordered complex is, by definition, a simplicial complex endowed with a total order relation on the set of its vertices. For instance, a complex whose vertices are numbered is ordered by the natural order of $\mathbb{N}$. A subcomplex of an ordered complex will be endowed with the order induced by restriction. In particular, a $k$-dimensional simplex $\Delta$ of $P$ will be considered as a $(k+1)$ uplet $\left(v_{0}, v_{1}, \ldots, v_{k}\right)$ with $v_{0}<v_{1}<\cdots<v_{k}$. Note that the order induced by restriction is compatible with the inclusion. More precisely, let $P^{\prime \prime} \subset P^{\prime} \subset P$ 
be subcomplexes, the order on $P^{\prime \prime}$ induced by the inclusion $P^{\prime \prime} \subset P^{\prime}$ is the same as the one induced by $P^{\prime \prime} \subset P$.

\subsection{Extension criterion.}

In this section, we state an extension criterion for maps defined on the vertices of simplicial complexes. We also treat the case where the extension is prescribed on a subcomplex.

Let $P$ be a locally finite $n$-dimensional simplicial complex and $f: P^{0} \longrightarrow$ $X$ be a map from the 0 -skeleton $P^{0}$ of $P$ to $X$. Fix $\alpha_{n}=\frac{1}{4.3^{n-1}-1}$.

Proposition 1.1. If the distance between the images of any pair of adjacent vertices is less than $\rho$ with $\rho<\alpha_{n} \kappa_{0}$, then $f$ extends to a continuous map $F: P \longrightarrow X$. Furthermore, the diameter of the image by $F$ of any simplex of $P$ is less than $\rho_{n}:=2.3^{n} \rho$.

Proof. Let us number the vertices of $P$ by natural integers and consider the induced order. Every $(k+1)$-dimensional simplex $\Delta^{k+1}$ of $P$ is endowed with the order induced by restriction and identifies in a natural way with the $(k+1)$-dimensional standard ordered simplex $\Delta_{0}^{k+1}=\left(x_{0}, \ldots, x_{k+1}\right)$. The points $x_{i}$ represent the vertices of the simplex.

The construction of the extension $F: P \longrightarrow X$ carries out successively on the skeleton of $P$. We will denote by $\varphi: \partial \Delta^{k+1} \longrightarrow X$ the restriction of $F$ to the boundary $\partial \Delta^{k+1}$ of a $(k+1)$-dimensional simplex $\Delta^{k+1}$ of $P$. The simplex $\Delta_{I}^{p}=\left(x_{i_{0}}, \ldots, x_{i_{p}}\right) \simeq \Delta_{0}^{p}$, where $I=\left\{i_{0}, \ldots, i_{p}\right\}$ is a subset of $\{0, \ldots, k+1\}$ with $i_{0}<\cdots<i_{p}$ and $p \leq k$, represents a $p$-face of $\Delta_{0}^{k+1}$. It identifies in a natural way with the $p$-dimensional standard ordered simplex $\Delta_{0}^{p}$.

We define $F$ on the 1-skeleton of $P$ by mapping each edge of $P$ to a minimizing arc between the images of the endpoints by $f$. In particular, the lengths of the images of the edges of $P$ are bounded from above by $\rho$.

Suppose now that we have defined by induction an extension $F: P^{k} \longrightarrow$ $X$ of $f$ on the $k$-skeleton $P^{k}$ of $P$. We also suppose that the restrictions of $\varphi$ to the $k$-faces $\Delta_{I}^{k}$ of $\Delta_{0}^{k+1}$, noted $\varphi_{I, k}: \Delta_{I}^{k} \simeq \Delta_{0}^{k} \longrightarrow X$ or more generally $\psi: \Delta_{0}^{k} \simeq \Delta_{I}^{k} \longrightarrow X$, satisfy the extension condition $\left(\mathcal{E}_{k}\right)$ which follows:

Let $x \in \Delta_{0}^{k-1}=\left(x_{0}, \ldots, x_{k-1}\right) \subset \Delta_{0}^{k}$, we designate by $x_{k} x$ the ray of $\Delta_{0}^{k}$ emanating from $x_{k}$ and joining $\Delta_{0}^{k-1}$ at $x$. The images by $\psi$ of the rays $x_{k} x$ for $x$ lying in $\Delta_{0}^{k-1}$ are parametrized proportionally to arclength. They are given by induction on $k$ from the restriction of $\varphi$ to the 1-skeleton of $\partial \Delta_{0}^{k+1}$ in the following way: 
Given $z \in \Delta_{0}^{k-2}=\left(x_{0}, \ldots, x_{k-2}\right) \subset \Delta_{0}^{k}$, let $x$ be a point lying in the ray $x_{k-1} z$ contained in $\Delta_{0}^{k-1}$ arising from $x_{k-1}$ and joining $z$ (see figure). This ray decomposes into three segments $z z^{\prime}, z^{\prime} z^{\prime \prime}$ and $z^{\prime \prime} x_{k-1}$ of equal lengths.

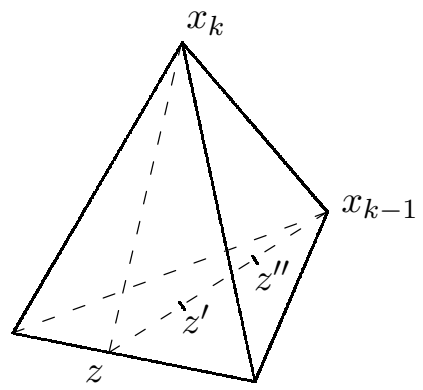

For any given $x$ running through $z z^{\prime}$, there exists a point $w$ in $z x_{k-1}$ which divides $z x_{k-1}$ into the same ratio as $x$ divides $z z^{\prime}$. The map $\psi$ takes then the ray $x_{k} x$ to the path $\psi\left(x_{k} z\right) \cup \psi(z w) \cup \psi(w x)$ where $\psi\left(x_{k} z\right), \psi(z w)$ and $\psi(w x)$ are given by induction as images of segments lying in some $(k-1)$ faces of $\Delta_{0}^{k-1}$.

Similarly, for any given $x$ running through $z^{\prime} z^{\prime \prime}$, there exists a point $w$ in $x_{k-1} x_{k}$ which divides $x_{k-1} x_{k}$ into the same ratio as $x$ divides $z^{\prime} z^{\prime \prime}$. The map $\psi$ takes the ray $x_{k} x$ to the path $\psi\left(x_{k} z\right) \cup \psi\left(z x_{k-1}\right) \cup \psi\left(x_{k-1} w\right) \cup \psi\left(w x_{k-1}\right) \cup$ $\psi\left(x_{k-1} x\right)$.

For $x=z^{\prime \prime}$, we get the path $\gamma \cup \psi\left(x_{k} x_{k-1}\right) \cup \psi\left(x_{k-1} z^{\prime \prime}\right)$ where $\gamma$ is a loop based at $\psi\left(x_{k}\right)$, image by $\psi$ of the triangle $x_{k} z x_{k-1}$ of sides $x_{k} z, z x_{k-1}$ and $x_{k-1} x_{k}$.

For $x$ dividing $z^{\prime \prime} x_{k-1}$ into a ratio $t$, the ray $x_{k} x$ is sent by $\psi$ to a path $\gamma_{t} \cup \psi\left(x_{k} x_{k-1}\right) \cup \psi\left(x_{k-1} x\right)$ where $\gamma_{t}$ is a homotopy of loops based at $\psi\left(x_{k}\right)$ with $\gamma_{0}=\gamma$ and $\gamma_{1}$ is the constant loop $\psi\left(x_{k}\right)$. Note that the loops $\gamma$ and $\gamma_{t}$ lying in the image of $\Delta_{0}^{k}$ are continuously parametrized by $z$ and will therefore be noted $\gamma_{\Delta_{I}^{k}, z}$ and $\gamma_{\Delta_{I}^{k}, z, t}$, or more simply $\gamma_{z}$ and $\gamma_{z, t}$.

Thus, the images of the rays $x_{k} x$, where $x$ runs along $x_{k-1} z$, give rise to a continuous family of loops $\gamma_{z}$ which contract into $\psi\left(x_{k}\right)$ through a continuous family of homotopies of loops $\gamma_{z, t}$ based at $\psi\left(x_{k}\right)$. Note that, reciprocally, such a family of homotopies $\gamma_{z, t}$ permits us to define the images of the rays $x_{k} x$ where $x$ runs along $x_{k-1} z$ using the restriction of $\varphi$ to the $(k-1)$-faces of $\partial \Delta_{0}^{k}$.

The maps $\psi$ are thus defined by induction from the restriction of $\varphi$ to the 1-skeleton of $\partial \Delta_{0}^{k+1}$ and some continuous families of homotopies $\gamma_{z, t}$. Furthermore, we assume that the images by $\psi$ of the rays $x_{k} x$ where $x$ lies in $\Delta_{0}^{k-1}$ are of length bounded from above by $\ell_{k}:=\left(2.3^{k-1}-1\right) \rho$. 
This concludes the statement of the extension condition $\left(\mathcal{E}_{k}\right)$.

Now, we want to define an extension $\Phi$ of $\varphi$ to $\Delta_{0}^{k+1}$ as a cone over $\Delta_{0}^{k}=$ $\left(x_{0}, \ldots, x_{k}\right)$ based at $x_{k+1}$, which satisfies the extension condition $\left(\mathcal{E}_{k+1}\right)$.

Let $x \in \Delta_{0}^{k}$. The ray of $\Delta_{0}^{k+1}$ emanating from $x_{k+1}$ and joining $x$ is noted $x_{k+1} x$. By definition, the images by the extension $\Phi$ of the rays $x_{k+1} x$ where $x$ lies in $\Delta_{0}^{k}$ are parametrized proportionally to arclength.

Let $z$ be the base point in $\Delta_{0}^{k-1}$ of the ray of $\Delta_{0}^{k}$ arising from $x_{k}$ and passing through $x$ where $x \in \Delta_{0}^{k}, x \neq x_{k}$. The image by $\varphi$ of the triangle $x_{k+1} z x_{k}$ of $\Delta_{0}^{k+1}$ of sides $x_{k+1} z, z x_{k}$ and $x_{k} x_{k+1}$ is noted $\gamma_{z}$.

We want to define the images by $\Phi$ of the rays arising from $x_{k+1}$ in the same manner as the restrictions $\psi$ of $\varphi$ to the faces of $\partial \Delta_{0}^{k+1}$ are defined (see above). For that, we need to construct a continuous family of homotopies $\gamma_{z, t}$ as it arises in the extension condition $\left(\mathcal{E}_{k}\right)$.

This family must be defined for $(z, t) \in \Delta_{0}^{k-1} \times[0,1]$. It must also contract the loops $\gamma_{z, 0}=\gamma_{z}$ based at $\psi\left(x_{k+1}\right)$ into the constant loops $\gamma_{z, 1} \equiv \psi\left(x_{k+1}\right)$ through a homotopy of loops $\gamma_{z, t}$ based at $\psi\left(x_{k+1}\right)$.

Since $\Phi$ extends $\varphi$, the images by $\Phi$ of the rays $x_{k+1} x$ where $x \in \partial \Delta_{0}^{k}$ are given by $\varphi$. Therefore, the family $\gamma_{z, t}$ with $(z, t) \in \partial \Delta_{0}^{k-1} \times[0,1]$ must agree with the homotopies $\gamma_{\Delta, z, t}$ where $\Delta$ is a $k$-face of $\partial \Delta_{0}^{k+1}$ containing $x_{k}$ and $x_{k+1}$.

The family of homotopies $\gamma_{z, t}$ is thus defined for $(z, t) \in \Delta_{0}^{k-1} \times \partial[0,1] \cup$ $\partial \Delta_{0}^{k-1} \times[0,1]=: \Sigma^{k-1} \simeq S^{k-1}$. By construction, the lengths of the loops $\gamma_{z, t}$ for $(z, t) \in \Sigma^{k-1}$ are bounded from above by $2 \ell_{k}+\rho<\kappa_{0}$. Therefore, the family $\gamma_{z, t}$ given by a map $\Sigma^{k-1} \longrightarrow \Omega_{x_{k+1}}^{2 \ell_{k}+\rho} X$ extends to $\Sigma^{k-1} \times[0,1] / \Sigma^{k-1} \times\{1\} \simeq \Delta_{0}^{k-1} \times[0,1] / \Delta_{0}^{k-1} \times\{1\} \simeq B^{k}$ with $L\left(\gamma_{z, t}\right) \leq 2 \ell_{k}+\rho$. Thus, the family $\gamma_{z, t}$ extends to $\Delta_{0}^{k-1} \times[0,1]$ and satisfies all the required conditions.

That completes the construction of the family of homotopies $\gamma_{z, t}$ and yields an extension $\Phi: \Delta_{0}^{k+1} \longrightarrow X$ of $\varphi$. By definition, $L\left(\Phi\left(x_{k+1} x\right)\right) \leq$ $3 \ell_{k}+2 \rho=\ell_{k+1}$ where $x$ lies in $\Delta_{0}^{k}$. Therefore, the map $F: P^{k} \longrightarrow X$ extends to the $(k+1)$-skeleton of $P$ into a map, still noted $F$, which satisfies the extension condition $\left(\mathcal{E}_{k+1}\right)$. We conclude then by induction.

The last statement of the proposition comes from the construction of $F$.

Remark. In order to extend $\varphi: \partial \Delta^{k+1} \longrightarrow X$ to $\Delta^{k+1}$, we only used the fact that the restrictions of $\varphi$ to the $k$-faces containing the vertex $x_{k+1}$ satisfy the extension condition $\left(\mathcal{E}_{k}\right)$ and that the length of the $\varphi$-images of the rays $x_{k} x$ lying in $\Delta_{0}^{k}=\left(x_{0}, \ldots, x_{k}\right)$ are bounded from above by $\ell_{k}$. We will use 
this remark afterwards in the following manner.

Given a subcomplex $Q$ of $P$, we number the vertices of $P$ starting by those of $Q$ and consider the induced order. The identification of the simplices of $Q$ with the standard simplex yields a natural metric length structure on $Q$.

Let $f: P^{0} \cup Q \longrightarrow X$ be a map and $\varepsilon>0$. Assume that the restrictions of $f$ to the simplices of $Q$ are $\varepsilon$-Lipschitz and that the restriction of $f$ to $P^{0}$ fulfills the hypothesis of Proposition 1.1. If $\varepsilon$ is small enough then the construction of the proof of Proposition 1.1 applies with the previously defined order on $P$, from the remark above. The map $f$ extends then to a continuous map $F: P \longrightarrow X$ as in the proposition.

\subsection{Volume and the shortest geodesic loop.}

Theorem A is established and the example of Riemannian homogeneous manifolds is presented in this section.

Proof of Theorem A. In order to apply R. Greene and P. Petersen's theorem (see [6, Theorem 1]) to derive a local volume estimate, we need to show that $M$ satisfies a condition of local geometric contractibility (see [6, p. 274]).

Fix $x_{0} \in M$ and $0<r<\alpha_{n} \operatorname{sgl}(M)$. We want to show that the ball $B\left(x_{0}, r\right)$ is contractible in $B\left(x_{0}, 4.3^{n} r\right)$. Without loss of generality, we can assume that the sphere $S\left(x_{0}, r\right)$ is represented by a finite $(n-1)$-dimensional simplicial complex $\sigma: Q \longrightarrow M$. The identification of the simplices of $Q$ with the standard simplex yields a natural metric length structure on $Q$. Since the map $\sigma: Q \longrightarrow M$ can be approximated by continuous maps homotopic to $\sigma$ whose restrictions to the simplices of $Q$ are Lipschitz, we can assume that the same holds for $\sigma$. Every simplicial complex endowed with the coherent topology admits a subdivision finer than a given open covering $\mathcal{U}$ (see [15, p. 126]). Fix $\varepsilon>0$, we apply this result to the open covering $\mathcal{U}_{\varepsilon}$ of $P$ where $\mathcal{U}_{\varepsilon}=\left\{U \subset Q \mid U\right.$ open, $f_{\mid U}$ is $k$-Lipschitz and $\operatorname{Diam}(U \cap Q) \leq$ $\frac{\varepsilon}{k}$ for some $\left.k>0\right\}$. Then we dilate the metric on $Q$ so that every simplex of the new triangulation of $Q$ is isometric to the standard simplex. The map $\sigma$ is $\varepsilon$-Lipschitz with respect to this new metric on $Q$.

Let $P$ be the cone over $Q$. We extend $\sigma$ to $P^{0} \cup Q$ by sending the vertex of $P \backslash Q$ to $x_{0}$. From the remark following Proposition 1.1, the map $\sigma$ extends to $\bar{\sigma}: P \longrightarrow B\left(x_{0}, 4.3^{n} r\right)$. Therefore, the ball $B\left(x_{0}, r\right)$ is contractible in $B\left(x_{0}, 4.3^{n} r\right)$. Thus, the continuous function $\rho:\left[0, \operatorname{sgl}(M)\left[\longrightarrow \mathbb{R}^{+}\right.\right.$is a local geometric contractibility function for $M$.

Using Theorem 1 of [6], we can conclude that there exists $C_{n}^{\prime}>0$ such that $\operatorname{Vol} B(R) \geq C_{n}^{\prime} R^{n}$ for every ball $B(R)$ of radius $R$ in $M$. 
The following well-known statement refines Theorem A for Riemannian homogeneous manifolds.

Lemma 1.2. The geodesic loops of Riemannian homogeneous manifolds agree with the simple closed geodesics.

Proof. Given a geodesic loop $\gamma$ based at $x_{0} \in M$, the first variation formula shows that $\frac{d}{d t} L\left(\gamma_{t}\right)=g\left(\dot{\gamma}(1), X\left(x_{0}\right)\right)-g\left(\dot{\gamma}(0), X\left(x_{0}\right)\right)=0$ where $\gamma_{t}$ is the variation of $\gamma$ induced by the Killing vector field $X$. Since the Killing vector fields generate the tangent space of homogeneous Riemannian manifolds, we have $\dot{\gamma}(1)=\dot{\gamma}(0)$. Therefore, $\gamma$ is closed.

As a corollary, there exists $C_{n}>0$ such that

$$
\operatorname{Vol}(M) \geq C_{n} \operatorname{scg}(M)^{n}
$$

for every Riemannian homogeneous manifold. This inequality contrasts with the case of the multi-dimensional systoles. Indeed, M. Katz constructs, in appendix D of [8], a sequence of homogeneous metrics on $S^{3} \times S^{3}$ such that $\frac{\operatorname{Vol}\left(g_{n}\right)}{\operatorname{sys}_{3}\left(g_{n}\right)^{2}} \rightarrow 0$, where $\operatorname{sys}_{3}(g)=\inf \left\{\operatorname{Vol}_{g}(S) \mid S\right.$ is a 3 -cycle such that $[S] \neq$ $\left.0 \in H_{3}\left(S^{3} \times S^{3}, \mathbb{Z}\right)\right\}$.

\section{Volume and short geodesic loops.}

In this part, we first present some filling estimates. Then, we define a functional on the double loop space and study the structure of some of its critical points. Finally, the proof of Theorem B is presented.

\subsection{Generalities.}

In this section, we recall the definition of the filling radius introduced by $\mathrm{M}$. Gromov in [7] and state general filling estimates.

Let $M$ be a complete Riemannian manifold and $L^{\infty}(M)$ be the space of all Borel functions on $M$ with the sup-norm. Because of the possible noncompactness of $M$, we consider unbounded functions on $M$; therefore, the distance between two of them may be infinite. However the natural embedding $i: M \hookrightarrow L^{\infty}(M)$ defined by $i(x)()=.\operatorname{dist}_{M}(x,$.$) is an isometry$ between metric spaces. Considering $M$ isometrically embedded in $L^{\infty}(M)$, we define $U_{r}(M)$ as the $r$-tubular neighborhood of $M$ in $L^{\infty}(M)$. 
Definition. The filling radius of $M$, denoted FillRad $(M)$, is the infinimum of positive reals $r$ such that there exists a locally finite $(n+1)$ dimensional chain $c$ in $U_{r}(M)$ whose boundary $\partial c$, contained in $M$, represents the fundamental class of $M$ in $H_{n}(M)$. That means the homomorphism $H_{n}(M) \longrightarrow H_{n}\left(U_{r}(M)\right)$ induced by the inclusion map vanishes. Here, the homology is with noncompact supports and the coefficients are in $\mathbb{Z}$ if $M$ is orientable and in $\mathbb{Z}_{2}$ otherwise.

The two following results, which provide upper bounds on the filling radius of Riemannian manifolds, will permit us to derive Theorem B. The first one was established by M. Gromov in [7, p. 41].

Theorem 2.1. Let $M$ be a complete Riemannian manifold of dimension $n$, then

$$
\operatorname{FillRad}(M) \leq c_{n} \operatorname{Vol}(M)^{\frac{1}{n}}
$$

where $c_{n}$ is a positive constant depending only on the dimension of $M$.

And the second one was proved by M. Katz in [9].

Theorem 2.2. Let $M$ be a closed Riemannian manifold of diameter $\operatorname{Diam}(M)$, then

$$
\operatorname{FillRad}(M) \leq \frac{1}{3} \operatorname{Diam}(M)
$$

\subsection{Morse theory on the double loop space.}

In this section, we define the double loop space, introduce a functional on it and describe one of its critical points.

Let $M$ be a complete Riemannian manifold. All the functional spaces will be endowed with the $C^{0}$-topology. Let $\mathcal{C}$ be the space of piecewise smooth curves $\gamma:[0,1] \longrightarrow M$. We recall that $\Lambda M$ and $\Omega M$ designate respectively the free loop space and the pointed loop space. The spaces $\Gamma^{\prime}=\left\{\Phi=\left(\gamma_{1}, \gamma_{2}\right) \in \mathcal{C} \times \mathcal{C} \mid \gamma_{1}(0)=\gamma_{1}(1)\right.$ and $\left.\gamma_{2}(0)=\gamma_{2}(1)\right\}$ and $\Gamma^{\prime \prime}=$ $\left\{\Phi=\left(\gamma_{1}, \gamma_{2}\right) \in \mathcal{C} \times \mathcal{C} \mid \gamma_{1}(0)=\gamma_{2}(1)\right.$ and $\left.\gamma_{1}(1)=\gamma_{2}(0)\right\}$ identify naturally with $\Lambda M \times \Lambda M$ and $\Lambda M$. Let $\Gamma$ be the union of $\Gamma^{\prime}$ and $\Gamma^{\prime \prime}$ and $\Gamma^{0} \subset \Gamma$ be the set of point curves. The intersection of $\Gamma^{\prime}$ and $\Gamma^{\prime \prime}$, noted $X$, represents the double loop space.

Next proposition will not be used afterwards. We state it because, describing the relative homotopy of $\Gamma$, it sets up the basis of a variational calculus on the double loop space. 
Proposition 2.3. Let $M$ be a simply connected closed manifold. Then, $\pi_{1}\left(\Gamma, \Gamma^{0}\right)$ is naturally isomorphic to $\pi_{2}(M)$.

Proof. Let us consider the natural projection

$$
\begin{aligned}
p: \Gamma & \longrightarrow M \times M \\
\Phi & \longmapsto\left(\gamma_{1}(0), \gamma_{2}(0)\right)
\end{aligned}
$$

This projection gives rise to two Serre fibrations with fiber $\Omega M \times \Omega M$ :

$$
p^{\prime}: \Gamma^{\prime}=\Lambda M \times \Lambda M \longrightarrow M \times M \text { and } p^{\prime \prime}: \Gamma^{\prime \prime}=\Lambda M \longrightarrow M \times M .
$$

Let $\Delta \simeq M$ be the diagonal of $M \times M$. We have $X=p^{-1}(\Delta)=$ $\Lambda M \times \Lambda M \cap \Lambda M$ and $p: X=\Gamma^{\prime} \cap \Gamma^{\prime \prime} \longrightarrow \Delta$ is the restricted fibration. The space $\Gamma$ may be written as $\Lambda M \times \Lambda M \amalg_{X} \Lambda M$. From Van Kampen's theorem, $\pi_{1}(\Gamma)$ is naturally isomorphic to $\pi_{1}(\Lambda M \times \Lambda M) *_{\pi_{1}(X)} \pi_{1}(\Lambda M)$. Since $M$ is simply connected, the exactness of the homotopy sequences of the fibrations implies that the groups $\pi_{1}(\Lambda M \times \Lambda M)$ and $\pi_{1}(X)$ are isomorphic to $\pi_{1}(\Lambda M) \oplus \pi_{1}(\Lambda M)$. Furthermore, the inclusion $X \hookrightarrow \Lambda M \times \Lambda M$ induces an isomorphism $\pi_{1}(X) \longrightarrow \pi_{1}(\Lambda M \times \Lambda M)$. Therefore, $\pi_{1}(\Gamma)$ is naturally isomorphic to $\pi_{1}(\Lambda M)$. Since $M$ is simply connected, $\pi_{1}(\Gamma)$ and $\pi_{1}(\Lambda M)$ are respectively isomorphic to $\pi_{1}\left(\Gamma, \Gamma^{0}\right)$ and $\pi_{2}(M)$. Hence the result.

We introduce the functional $\mathcal{L}$ on $\Gamma$ defined by $\mathcal{L}(\Phi)=\max \left\{L\left(\gamma_{1}\right), L\left(\gamma_{2}\right)\right\}$ where $\Phi=\left(\gamma_{1}, \gamma_{2}\right) \in \Gamma$. The restriction of $\mathcal{L}$ to $X$ induces a functional $\mathcal{L}_{X}$ on the double loop space. Let $L_{0}(M)$ be the infinimum of the positive local minima of $\mathcal{L}_{X}$.

The following lemma describes the structure of the local minima of $\mathcal{L}_{X}$.

Lemma 2.4. Let $\Phi \in X$ be a positive local minimum of $\mathcal{L}_{X}$. Then, $\Phi$ is formed either of a nontrivial closed geodesic to which a loop is attached or of two distinct nontrivial geodesic loops $\gamma_{1}$ and $\gamma_{2}$ of the same length based at the same point. In the second case, the differences $v_{1}$ and $v_{2}$ of the tangent vectors at the endpoints of $\gamma_{1}$ and $\gamma_{2}$ point to different directions. That is $g\left(v_{1}, v_{2}\right) \leq 0$ where $v_{i}=\dot{\gamma}_{i}(1)-\dot{\gamma}_{i}(0)$ is different from 0 .

Proof. Let $\gamma_{1}$ and $\gamma_{2}$ be the two loops forming $\Phi$. Suppose that none of them is a closed geodesic of null index. In this case, the loops $\gamma_{i}$ are homotopic to a shorter closed curve. If $L\left(\gamma_{1}\right)>L\left(\gamma_{2}\right)$ then there exists a $\mathcal{L}_{X}$-decreasing deformation of $\Phi$ (which possibly slightly increase the length of $\gamma_{2}$ ). This is 
impossible because $\Phi$ is a local minimum of $\mathcal{L}_{X}$. Switching the roles of $\gamma_{1}$ and $\gamma_{2}$, we get $L\left(\gamma_{1}\right)=L\left(\gamma_{2}\right)$. The loops $\gamma_{1}$ and $\gamma_{2}$ are geodesic, otherwise the Birkhoff curve-shortening flow with fixed endpoints would decrease the length of one of them. This would contradict the inequality $L\left(\gamma_{1}\right)=L\left(\gamma_{2}\right)$ for the local minima of $\mathcal{L}_{X}$. If $g\left(v_{1}, v_{2}\right)>0$, the first variation formula shows that it would be possible to decrease simultanuously the lengths of $\gamma_{1}$ and $\gamma_{2}$ through a local flow. Hence another contradiction.

Remark. When $M$ is compact, $L_{0}(M)$ is positive. Furthermore, $L_{0}(M)$ is reached either by the length of a nontrivial closed geodesic of null index or by half the length of a geodesic double loop formed of two distinct nontrivial geodesic loops of the same length based at the same point. Therefore, we have $\ell_{0}(M) \leq 2 \min \left\{\operatorname{scg}(M), L_{0}(M)\right\}$.

\subsection{Volume and double geodesic loops.}

Now, we derive Theorem B from Theorems 2.1 and 2.2, the previous remark and the following inequality.

Proposition 2.5. Let $M$ be a 2-essential complete Riemannian manifold, then

$$
\operatorname{FillRad}(M) \geq \frac{1}{24} \ell_{0}(M)
$$

Proof. By definition, the fundamental class $[M]$ of $M$ vanishes in $U_{\delta}(M) \subset$ $L^{\infty}(M)$ where $\delta>\operatorname{FillRad}(M)$. Therefore, there exists a continuous map $\tilde{\sigma}: P \longrightarrow U_{\delta}(M)$ from an $(n+1)$-dimensional simplicial complex $P$ such that the restriction $\sigma: \partial P \longrightarrow M$ represents $[M]$ in $H_{n}(M)$. Since $M$ is 2-essential, there exists a continuous map $\psi: M \longrightarrow K$ into a $K(\pi, 2)$ space, which induces a nontrivial morphism $\psi_{*}: H_{n}(M) \longrightarrow H_{n}(K)$. The image of $Q:=\partial P$ by $\psi \circ \sigma$ represents $\psi_{*}[M]$ in $H_{n}(K)$. Suppose that $\delta<\frac{1}{24} \ell_{0}(M)$. We are going to construct a continuous map $F: P \longrightarrow K$ which agrees with $\psi \circ \sigma$ on $\partial P$. This will lead to a contradiction since $\psi_{*}[M] \neq 0$. Therefore, $M$ does not bound in $U_{\delta}(M)$ with $\delta<\frac{1}{24} \ell_{0}(M)$, hence the inequality $\operatorname{FillRad}(M) \geq \frac{1}{24} \ell_{0}(M)$.

Subdividing $P$ if necessary, we can assume that the diameter of the image by $\tilde{\sigma}$ of the simplices of $P$ is less than $\varepsilon>0$ with $\varepsilon<\frac{1}{12} \ell_{0}(M)-2 \delta$. We first define a map $f: P^{0} \cup Q \longrightarrow M$ with $f_{\mid Q}=\sigma$ by sending each vertex $p_{i}$ of $P$ to a nearest point of $\tilde{\sigma}\left(p_{i}\right)$ in $M$, as we wish. Since $i: M \hookrightarrow U_{\delta}(M)$ is 
isometric, every pair $p_{i}, p_{j}$ of adjacent vertices of $P$ satisfies

$$
\begin{aligned}
d_{M}\left(f\left(p_{i}\right), f\left(p_{j}\right)\right) & \leq d_{L^{\infty}}\left(f\left(p_{i}\right), \tilde{\sigma}\left(p_{i}\right)\right)+d_{L^{\infty}}\left(\tilde{\sigma}\left(p_{i}\right), \tilde{\sigma}\left(p_{j}\right)\right)+d_{L^{\infty}}\left(\tilde{\sigma}\left(p_{j}\right), f\left(p_{j}\right)\right) \\
& \leq 2 \delta+\varepsilon=: \rho<\frac{1}{24} \ell_{0}(M)
\end{aligned}
$$

We extend the map $f$ to $P^{1}$ by taking the edges of $P$ to minimizing segments joining the image of their endpoints. Let $\Delta^{2}$ be a 2-simplex of $P$. By construction, the length of the image of $\partial \Delta^{2}$ is less than $3 \rho<\operatorname{scg}(M)$. Therefore, the Birkhoff curve-shortening process defines a map from the disk $D \simeq \Delta^{2}$ to $M$, which sends $\partial D=\partial \Delta^{2}$ onto its image. This construction yields an extension $f: P^{2} \cup Q \longrightarrow M$ of $\sigma$.

We want now to extend it to $P^{3}$. This is possible if the image of the boundary $\partial \Delta^{3}$ of every 3 -simplex $\Delta^{3}$ of $P$ bounds a ball in $M$. Let $\Delta_{i}$ be the faces of $\Delta^{3}$. The boundaries $\partial \Delta_{i}$ converge to point curves through homotopies $\left(c_{t}^{i}\right)_{0 \leq t \leq 1}$, given by the Birkhoff curve-shortening process, which define the images of $\Delta_{i}$. The curves $c_{0}^{i}$ agree with the images of $\partial \Delta_{i}$ by $f$ and the curves $c_{1}^{i}$ reduce to point curves. Note also that $L\left(c_{t}^{i}\right)<\operatorname{scg}(M)$. Using homotopies to reparametrize the loops if necessary, we can assume that $c_{0}^{1}(s)$ and $c_{0}^{2}(s)$ agree and run through their common edge for $\frac{2}{3} \leq s \leq 1$. The family $\Phi_{t}=\left(c_{1-t}^{1}, c_{1-t}^{2}\right) \in \Gamma^{\prime \prime}, 0 \leq t \leq 1$, extends to $\Gamma$ through the homotopy $\Phi_{t}(s)=\left(c_{0}^{1}\left(\frac{s}{t}\right), c_{0}^{2}\left(\frac{s}{t}\right)\right) \in \Gamma^{\prime}$ where $1 \leq t \leq \frac{3}{2}$ and $0 \leq s \leq 1$. The same goes for $c_{t}^{3}$ and $c_{t}^{4}$. This yields two homotopies of $\Gamma$. We use now homotopies to reparametrize the final curves so that they agree. Putting these two homotopies together gives rise to a one-parameter family of curves $\Phi_{t} \in \Gamma$, $0 \leq t \leq 3$, starting and ending at point curves.

Let us show now that the class it induces in $\pi_{1}\left(\Gamma, \Gamma^{0}\right)$ is trivial. The family decomposes into three parts :

- $\left(\Phi_{t}\right)_{0 \leq t \leq 1}$ and $\left(\Phi_{t}\right)_{2 \leq t \leq 3}$, which join in $\Gamma^{\prime \prime}$ point curves to double loops.

- $\left(\Phi_{t}\right)_{1 \leq t \leq 2}$, which join in $\Gamma^{\prime}$ the two double loops $\Phi_{1}$ and $\Phi_{2}$.

Since $\mathcal{L}\left(\Phi_{1}\right)$ and $\mathcal{L}\left(\Phi_{2}\right)$ are $<3 \rho<L_{0}(M)$, the two double loops $\Phi_{1}$ and $\Phi_{2}$ converge to point curves through $\mathcal{L}$-non-increasing homotopies $\Phi_{t}^{1}$ and $\Phi_{t}^{2}$ of $X$. In particular, each of the loops which compose them converge to point curves through homotopies given by $\Phi_{t}^{1}$ and $\Phi_{t}^{2}$. The length of the loops of these homotopies is less than $3 \rho<\operatorname{scg}(M)$. Therefore, they are homotopic to the $c_{t}^{i}$ s. Indeed, two homotopies starting from the same loop and ending at point curves form a path in $\Lambda M$ with endpoints in $\Lambda^{0} M$. Such a path composed of loops of length $<\operatorname{scg}(M)$ is homotopically trivial in $\pi_{1}\left(\Lambda M, \Lambda^{0} M\right)$ from the minimax principle of Fet and Lyusternik (see [10]). Since we want to show that the family $\Phi_{t}$ bounds in $M$, we can assume that 
$\left(\Phi_{t}\right)_{0 \leq t \leq 1}$ and $\left(\Phi_{t}\right)_{2 \leq t \leq 3}$ agree with $\Phi_{t}^{1}$ and $\Phi_{t}^{2}$. Under this assumption, $\Phi_{t}$ lies in $\Gamma^{\prime} \simeq \Lambda M$. It gives rise to a path in $\Lambda M$ which starts and ends at point curves with $L\left(\Phi_{t}\right)<6 \rho<\operatorname{scg}(M)$. As previously, this implies that this path is homotopically trivial. Therefore, the image of the boundary of every 3 -simplex of $P$ bounds a ball in $M$. The map $f$ extends to $P^{3}$.

From obstruction theory, the map $F: P^{3} \cup Q \stackrel{f}{\longrightarrow} M \stackrel{\psi}{\longrightarrow} K$ extends to $P$. Hence the result.

Remark. Generically, i.e., for a metric lying in a dense open set of the space of metrics endowed with the $C^{2}$ topology, closed geodesics are nondegenerate and have different lengths. This property does not hold for geodesic loops. It is not possible to get rid of the double loops formed of two distinct nontrivial geodesic loops of the same length by slightly perturbing the metric.

We only sketch the arguments. Let us consider a metric which has two distinct geodesic loops of the same length based at the same point $x_{0}$. We suppose that these geodesic loops are non-degenerate and that their index is null. This construction may be carried out by modifying the metric on a tubular neighborhood of a figure-eight curve. These two geodesic loops vary continuously with their base-point in the neighborhood of $x_{0}$. Locally, the set of base-points for which the two geodesic loops have equal length forms a hypersurface. This hypersurface is stable and does not disappear by slightly perturbing the metric. Therefore, on open sets of metrics, points do exist through which two geodesic loops of the same length pass. Given a metric with three distinct (non-degenerate) geodesic loops of the same length based at the same point, the previous arguments suggest that this triple loop configuration might disappear after some perturbations of the metric.

\section{References.}

[1] M. Anderson, Ricci curvature bounds and Einstein metrics on compact manifolds, J. Amer. Math. Soc. 2 (1989) 455-490.

[2] I. Babenko, Asymptotic invariants of smooth manifolds, Russian Acad. Sci. Izv. Math. 41 (1993) 1-38.

[3] M. Berger, Une borne inférieure pour le volume d'une variété riemannienne en fonction du rayon d'injectivité, Ann. Inst. Fourier, 30 (1980) 259-265. 
[4] C. Croke, Some isoperimetric inequalities and eigenvalue estimates, Ann. Sci. École Norm. Sup. 13 (1980) 519-535.

[5] C. Croke, Area and the length of the shortest closed geodesic, J. Differential Geometry 27 (1988) 1-21.

[6] R. Greene \& P. Petersen V, Little topology, big volume, Duke Math. J. 67 (1992) 273-290.

[7] M. Gromov, Filling Riemannian manifolds, J. Differential Geometry 18 (1983) 1-147.

[8] M. Gromov, Metric structures for Riemannian and non-Riemannian spaces, Progr. Math., Vol. 152, Birkhäuser, Boston, MA, 1998.

[9] M. Katz, The filling radius of two-point homogeneous spaces, J. Differential Geometry 18 (1983) 505-511.

[10] W. Klingenberg, Lectures on closed geodesics, Grundlehren Math. Wiss. 230, Springer, Berlin, 1978.

[11] A. Nabutovsky, R. Rotman, Upper bounds on the length of a shortest closed geodesic and quantitative Hurewicz theorem, J. Eur. Math. Soc. 5 (2003) 203-244.

[12] A. Nabutovsky, R. Rotman, Volume, diameter and the minimal mass of a stationary 1-cycle, preprint.

[13] P. Petersen V, A finiteness theorem for metric spaces, J. Differential Geometry 31 (1990) 387-395.

[14] R. Rotman, Upper bounds on the length of the shortest closed geodesic on simply connected manifolds, Math. Z. 233 (2000) 365-398.

[15] E. Spanier, Algebraic topology, Springer-Verlag, Berlin, 1995

DÉPARTEMEnT De Mathématiques

UNIVERSITÉ DE TOURS

PARC DE GRANDMONT

37200 Tours, France

sabourau@gargan.math.univ-tours.fr

Received December 17, 2002. 
